\title{
Virology and epidemiology analyses of global adenovirus-associated conjunctivitis outbreaks, 1953-2013
}

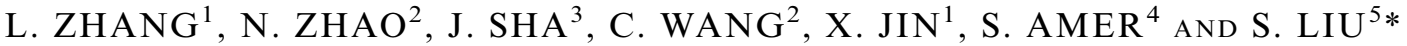 \\ ${ }^{1}$ Eye Center, Second Affiliated Hospital, Medical School of Zhejiang University, Hangzhou, Zhejiang Province, \\ China \\ ${ }^{2}$ National Research Center for Wildlife Born Diseases, Institute of Zoology, Chinese Academy of Sciences, \\ Beijing, China \\ ${ }^{3}$ Department of Gastroenterology, The 421 Hospital of Chinese People's Liberation Army, Guangzhou, \\ Guangdong Province, China \\ ${ }^{4}$ Department of Zoology, Faculty of Science, Kafr El Sheikh University, Kafr El Sheikh, Egypt \\ ${ }^{5}$ Department of Infectious Diseases, Zhejiang Provincial Center for Disease Control and Prevention, Hangzhou, \\ Zhejiang Province, China
}

Received 15 August 2015; Final revision 11 November 2015; Accepted 2 December 2015; first published online 6 January 2016

\section{SUMMARY}

This study aimed to compare the virology and epidemiology of epidemic keratoconjunctivitis (EKC), pharyngoconjunctival fever (PCF) and acute haemorrhagic conjunctivitis (AHC) outbreaks worldwide caused by the human adenovirus (HAdV) from 1953 to 2013. Eighty-three hexon sequences from 76 conjunctivitis outbreaks were analysed and subtyped using Mega 5.05, Clustal X and SimPlot software. Epidemiology was performed for the area, age and seasonal distribution. A phylogenetic analysis indicated that all the isolates could be divided into three subgenetic lineages, without a common ancestor. The major causes of the outbreaks were Ad8, Ad7 and Ad2 co-infection with enterovirus 70 (EV70) in EKC, PCF and AHC, respectively. The epidemiological findings suggested that $\mathrm{EKC}$ and $\mathrm{AHC}$ were circulating predominantly in Asia during the early winter and spring, whereas PCF was circulating mainly in China, Australia and the United States during the summer. This study suggests that EKC, AHC and PCF outbreaks have different circulating patterns throughout the world and are caused by different adenovirus serotypes. A global surveillance system should be established to monitor conjunctivitis outbreaks in the future.

Key words: Adenovirus, conjunctivitis, ocular infection, outbreak, virology.

\section{INTRODUCTION}

Adenoviruses are medium-sized $(90-100 \mathrm{~nm})$, non-enveloped, double-stranded DNA viruses that can infect a broad range of vertebrate hosts worldwide. At least 57 distinct serotypes of adenoviruses

\footnotetext{
* Author for correspondence: Dr S. Liu, Department of Infectious Diseases, Zhejiang Provincial Center for Disease Control and Prevention, 3399 Binsheng Road, Hangzhou, Zhejiang Province, 310051, China.

(Email: liushelan@126.com or liushelan@gmail.com)
}

and seven adenoviral species (A-G) have been detected in humans since they were first isolated in 1953 [1, 2]. Human adenoviruses (HAdVs) cause a wide range of illnesses, including ocular, respiratory and gastrointestinal infections, which vary in their severity, depending on the serotype [3-5]. HAdV subgroups B and D are mainly responsible for ocular infections, including epidemic keratoconjunctivitis (EKC), pharyngoconjunctival fever (PCF) and acute haemorrhagic conjunctivitis (AHC) [5-9]. The predominant serotypes differ among countries/regions 
and change over time. In recent years, the transmission of novel strains between countries or across continents, in addition to the replacement of dominant serotypes with new strains, has been reported. A series of adenovirus-related conjunctivitis outbreaks has been reported worldwide, with different epidemiological patterns.

Currently, there are no effective vaccines against ocular infections caused by adenoviruses [10]. To date, few countries, with the exception of Japan and the United States, have established adenovirus surveillance systems [11, 12]. The present study aimed to compare the epidemiological features and viral evolution of EKC, AHC and PFC outbreaks based on 83 adenovirus hexon sequences and 76 outbreaks in the past 60 years. This research will help to improve our current understanding of conjunctivitis and to establish a suitable strategy for the control and prevention of HAdV-associated ocular infection.

\section{METHODS}

\section{Outbreak data and hexon gene sources}

Epidemiological data on 76 adenovirus-associated ocular outbreaks (48 EKC, 17 PCF and 11 AHC outbreaks) from 1953 to 2013 were obtained from PubMed Central (http://www.ncbi.nlm.nih.gov/pubmed/), as well as from the website of the WHO (http://www.who.int/en). Eighty-three representative HAdV hexon sequences collected from ocular and respiratory outbreaks were retrieved from GenBank (https://www.ncbi.nlm.nih. gov/genbank/). Hexon sequences were selected using the following criteria: (1) sequences generated from low- and high-virulence/pathogenic strains; (2) sequences of representative strains responsible for outbreaks of ocular and respiratory infections; (3) sequences of different serotypes in different years; (4) sequences from high-circulating areas/countries and low-circulating areas/countries; (5) vaccine strains were contained in this study as the reference strains. The sequence accession numbers and genotypes are listed in Supplementary Table S1.

\section{Homology and phylogenetic tree of the hexon gene}

The sequences were aligned with Clustal X (ftp:// ftp-igbmc.u-trasbg.fr/pub/ClustalX/) to determine their identity or similarity. Phylogenetic analyses of the aligned sequences were inferred based on neighbour-joining and maximum-likelihood methods using Mega 5.05 (http://www.megasoftware.net) and PHYLIP v. 3.67 software (http://evolution.gs.wash ington.edu/phylip.html). The plot similarity vs. position and mosaic sites was performed with SimPlot v. 3.5.1 [13] (the Johns Hopkins School of Medicine, USA) to determine the degree of identity of the query sequence (HAdV-7 vaccine strain) to a panel of reference sequences comprised of 8 hexon sequences from ocular infections in a sliding window (windows 200 $\mathrm{bp} / \mathrm{step}$ ). To deduce the common ancestor, a hexon sequence network was constructed using Network v. 4.611 software (Fluxus Technology Ltd, UK).

\section{Structure modelling of hexon proteins}

Three protein query Hexon sequences, an EKC infection strain (AB330133), one respiratory infection strain (JF800905) [14] and a respiratory infection HAdV-7 vaccine strain (AY594256) [15], were uploaded to the SWISS-MODEL site (http://swissmodel.expasy. org/workspace/). The three-dimensional (3D) structure of hexon proteins was constructed based on the template 2OBE (1.45 $\AA$ ) (ID). A hexon sequence of a representative ocular infection strain was selected from an EKC outbreak of a high-prevalence area. The predicted protein structure of the strains was superimposed on that of the retrieved protein templates using Visual Molecular Dynamics v. 1.8.6 software [16].

\section{Statistical analysis}

The locations of the PCF and AHC outbreaks were geocoded by the Google Map geocoding service (https:// google-developers.appspot.com/maps/documentation/ javascript/examples/geocoding-simple), and the $\mathrm{x}$ (longitude) and y (latitude) coordinates were recorded. Then, ArcGIS (ArcMap, v. 10.2, ESRI Inc., USA) was used to generate a spatial distribution map.

\section{RESULTS}

\section{Hexon gene phylogeny and homology}

The homology analyses based on the alignment of hexon sequences from the HAdV-associated ocular infections showed that the ocular strains were closely related $(75 \cdot 3-100 \%$ homology) compared to 41 $50.3 \%$ similarity for those involved in respiratory infection. The phylogenetic analyses of the serotypes revealed an ocular HAdV cluster in the three subgenetic lineages that was very different from those 


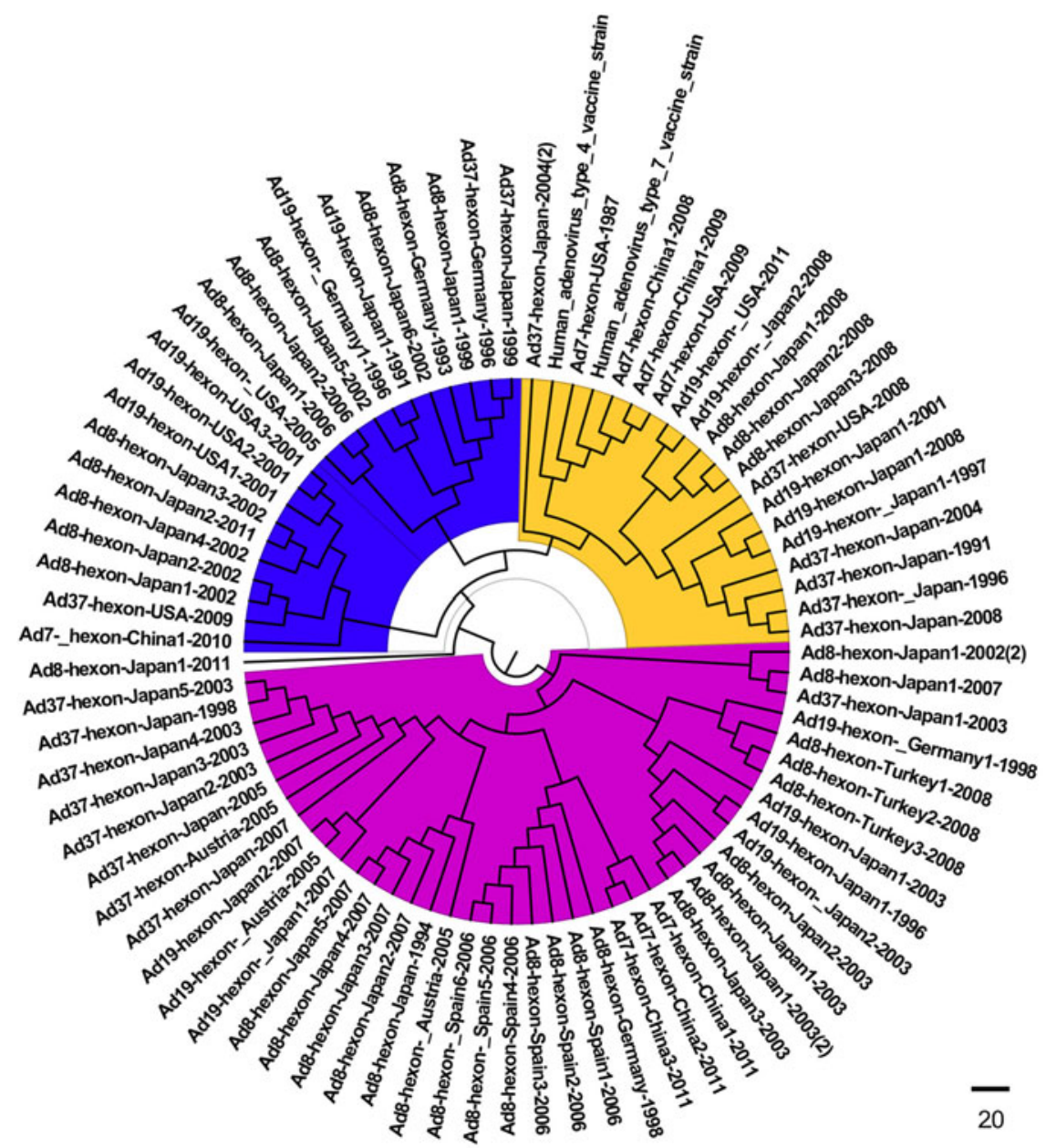

Fig. 1. Phylogenetic tree based on the hexon gene of HAdV strains isolated from ocular, respiratory and vaccine strains retrieved from GenBank, listed in Supplementary Table S1. Subgroup 1, blue; subgroup 2, yellow; subgroup 3, purple).

obtained from the lung (Fig. 1). The plot similarity $v$. position showed that the percentage of homology was $50-92 \%$ in position nucleotide (nt) $0-400,50-66 \%$ in position nt $400-800,50-84 \%$ in position nt $800-1200$, $50-86 \%$ in position nt $1200-2400$ and $66-90 \%$ in the positions after nt 2400 regions, respectively (Supplementary Fig. 1). In addition, nt 400-800 was highly diverse compared to that of the other regions. A median-joining network analysis showed that the $83 \mathrm{HAdV}$ isolates from the respiratory and ocular infections had no common ancestor and that they could be segregated into three subgroups. No geographical/regional clusters were identified in any of the sublineages in any of the years (Fig. 2, Supplementary Table S2).

\section{D structure prediction of the hexon protein}

To compare the structural modelling of the dominant neutralizing epitope functionally residing in the single capsid polypeptide (amino acids 103-111, 369-378, 589-600), representative isolates from the ocular (AB330133, Ad19), respiratory (F800905, Ad7) and vaccine (AY594254, Ad4) strains were analysed. The 3D structure showed that the major HAdV capsid protein (hexon) of the different strains all possessed a $\beta$-barrel structural motif, which included three variable loops, a silk-like random coil, helices, a folded sheet and a rolled angle. The same motif has been found in icosahedral capsids of diverse DNA viruses [2]. The dominant neutralizing epitope was located on the outer surface of the capsid in all three strains (a yellow loop indicates amino acids 103-111, a red loop indicates amino acids 369-378, and a green loop indicates amino acids 589-600). The configuration of the three loops in the respiratory strain and its vaccine strain was very similar, with the green and yellow loops inter-related together with the red loop appearing to be more separated. In contrast, 


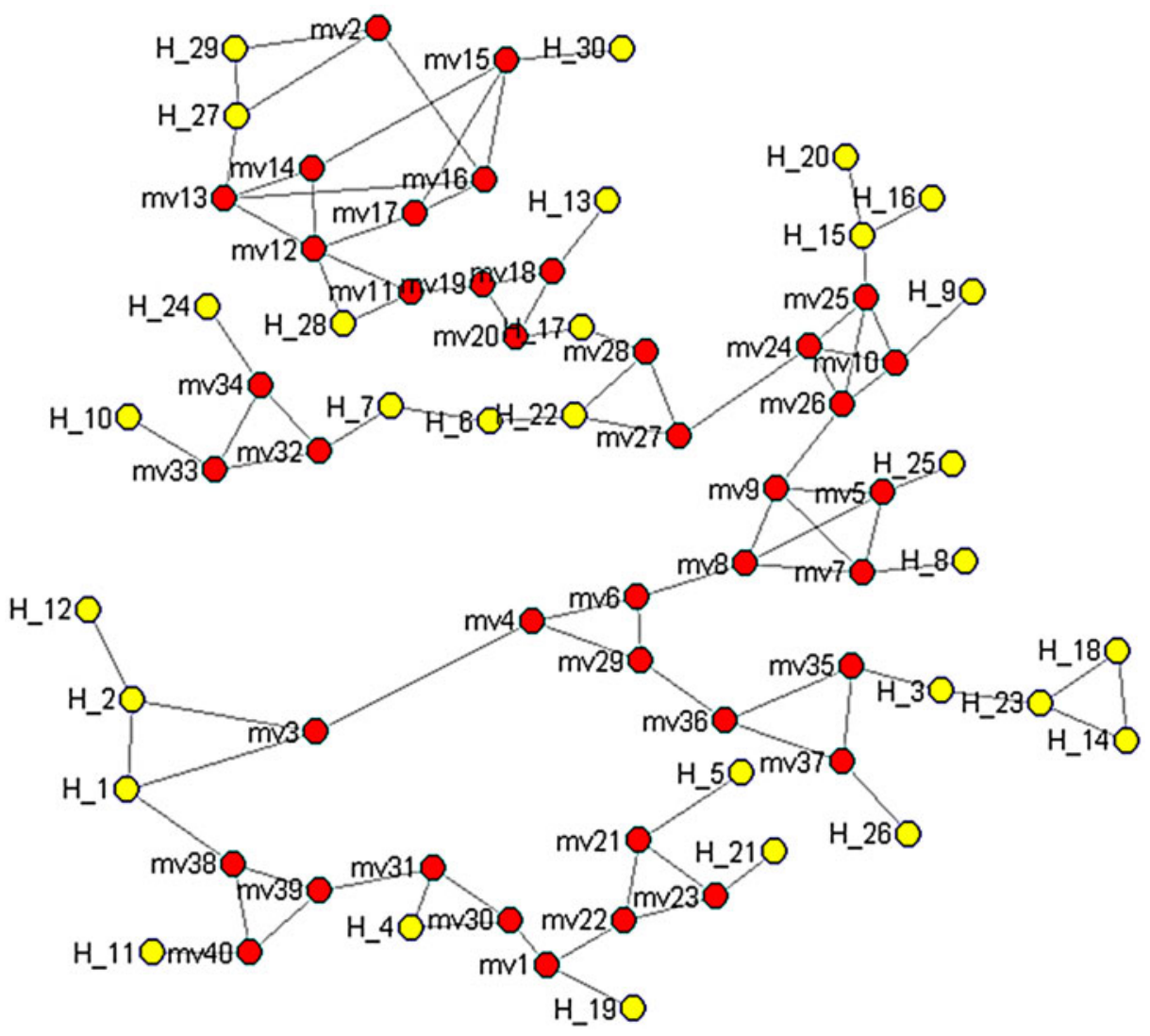

Fig. 2. Median-joining network depicting the relationships between the respiratory, ocular and vaccine strains between 1953 and 2013. The analysis is based on hexon gene sequences of 83 isolates deposited in GenBank from worldwide outbreaks between 1953 and 2013. The red points denote hereditary points. The yellow points denote hexon sequences. Information on the haplotype distribution is provided in Supplementary Table S2.

the three loops were significantly separated in the hexon protein from the ocular infection strain, where the red loops were the most important epitope (Fig. 3).

\section{Epidemiological features of the EKC, PCF and AHC outbreaks}

Forty-eight EKC outbreaks were reported worldwide, with most attributed to HAdV-8, $-19,-37,-53$ and -54 [17-60]. As shown in Figure 4a, EKC outbreaks were widely reported in Europe, including the UK, France, Spain, Turkey, Greece, Sweden, Russia and Germany, with HAdV-8 being the most prominent strain. EKC outbreaks were also reported in Mexico, Brazil and Chile in Latin America, as well as in the United States and Canada in North America. EKC outbreaks were also reported in Africa and Asia, including India, China and Japan. Different HAdV serotypes were isolated from various outbreaks. In 1953-1969, serotype HAdV-8 accounted for $100 \%$ of all outbreaks. In 1970-1979, HAdV-8, -4 and -19 accounted for
$44 \cdot 44 \%, 11 \cdot 11 \%$ and $4 \cdot 44 \%$, respectively, of all outbreaks. In 1980-1989, HAdV-8 accounted for $63.64 \%$ of outbreaks. HAdV-3, -37 and -7 were responsible for $18 \cdot 8 \%, 9 \cdot 09 \%$ and $9 \cdot 09 \%$ of all outbreaks, respectively, in the same period. In 1990 1999, HAdV-8 was responsible for $77 \cdot 27 \%$ of all outbreaks, and HAdV-29 and -37 were each responsible for $9 \cdot 66 \%$ of all outbreaks. In 2000-2009, HAdV-8 accounted for $84.62 \%$ of all outbreaks, whereas HAdV-54 and -4 were each responsible for $7 \cdot 69 \%$ of all outbreaks. Finally, in 2010-2013, HAdV-8 accounted for $66.67 \%$ of all outbreaks, and HAdV-2 accounted for $33.33 \%$ (Fig. $4 b$ ). The occurrence of the EKC outbreaks fluctuated over time. Of the reported outbreaks between 1953 and 2013, 8.33\% occurred before 1970. In the years 1970-1979, 1980 1989, 1990-1999, 2000-2009 and 2010-2013, the reported incidence was $18 \cdot 75 \%, 22.92 \%, 16 \cdot 67 \%$, $27 \cdot 08 \%$ and $6 \cdot 25 \%$, respectively. Public facilities, especially those related to the healthcare sector, played a pivotal role in the EKC outbreaks. Ophthalmology 

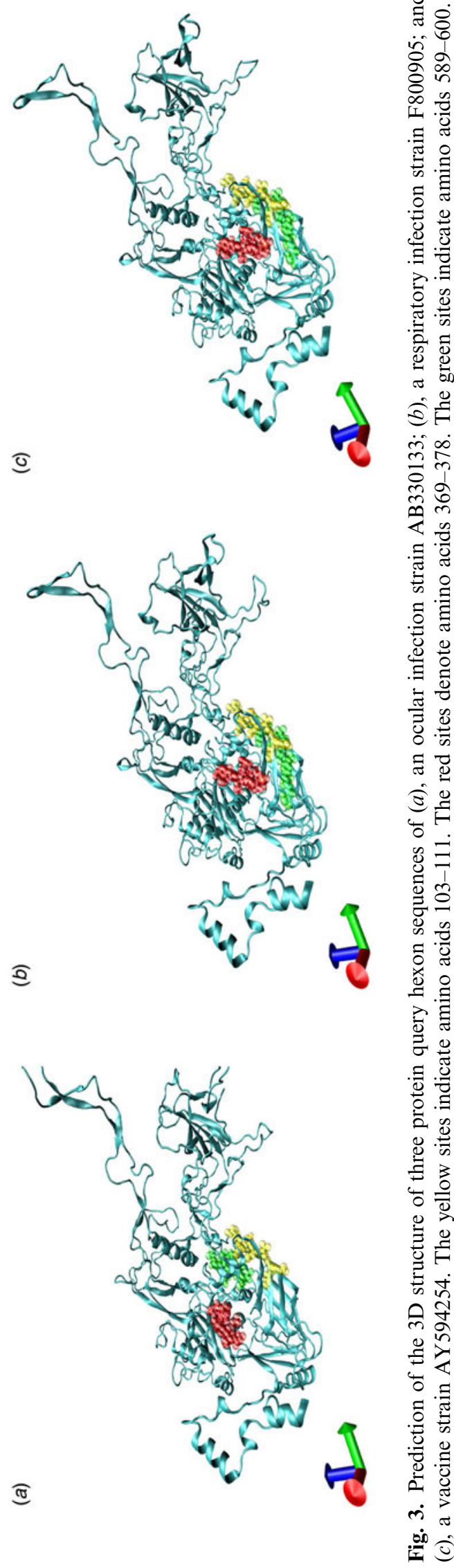

clinics were the most frequent sources of EKC and accounted for $65 \cdot 22 \%$ of all outbreaks. Neonatal intensive care units (ICUs) accounted for $8.70 \%$ of infections, and infant daycare centres and multidisciplinary hospitals each accounted for $6 \cdot 52 \%$. Geriatric centres, community centres, industrial facilities and nursery schools also contributed to the EKC outbreaks (Fig. 5a). Figure $5 b$ shows the outbreaks were reported in different population sizes. In most of the EKC outbreaks, fewer than 100 people were infected. All age groups (4-85 years) were vulnerable to EKC infections during the outbreaks (data not shown).

From 1953 to 2013, 17 large-scale PFC outbreaks were reported from different areas worldwide (Table 1). Most of these were caused by HAdV-3, $-4,-7,-8$ and -19 , with HAdV-3 being the predominant strain. The positive rate ranged from $32.0 \%$ to $100 \%$ in the PFC outbreak samples. In 11 of 17 outbreaks, the outbreak usually infected a population size under 100. The distribution of the serotypes varied at different times and in different locations. With regard to the timing of the PFC outbreaks between 1953 and 2013, 5.88\% were reported before 1970 . The reported incidence in 1970-1979, 1980-1989, 1990-1999, 2000-2009 and 2010-2013 was $23 \cdot 53 \%$, $17 \cdot 65 \%, 17 \cdot 65 \%, 23 \cdot 53 \%$ and $11 \cdot 76 \%$, respectively. Swimming pools were the most common source of the outbreaks. Summer camps were also implicated. PCF most commonly affected older teenagers.

Since the first description of an AHC outbreak in Ghana in 1969 [61], 11 outbreaks caused by the adenovirus have been reported. The main serotypes were HAdV-2, -4, -7, -8, -11 and -19. On average, 50 patients were affected in each outbreak. There was no predominant isolate and significant epidemical locations distribution. Interestingly, nine AHC outbreaks were associated with a concurrent infection, including picornavirus, EV70, coxsackievirus, herpes simplex virus (HSV) and chlamydia. The case-positive rate ranged from $8.33 \%$ to $53.33 \%$ in the AHC outbreak samples (Table 2). Similar to EKC, the AHC outbreaks were mostly linked to ophthalmology clinics, hospitals and crowded facilities, and the most vulnerable populations were those aged 15-40 years (Table 2).

\section{DISCUSSION}

Although previous studies have described adenovirus outbreaks associated with respiratory infections 

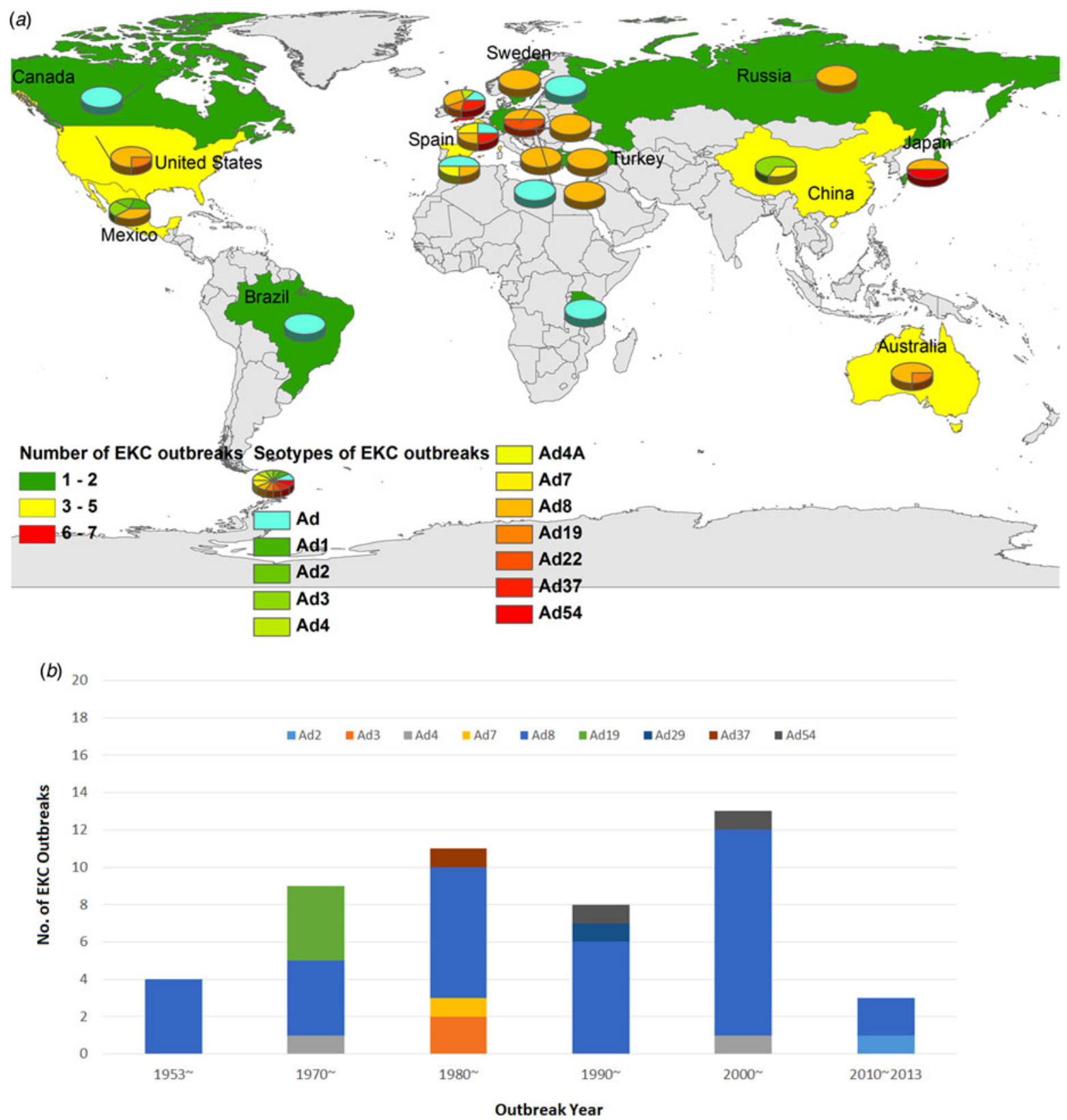

Fig. 4. The geographical distribution (a) and timeline (b) of the $48 \mathrm{EKC}$ adenovirus outbreaks from 1953 to 2013 worldwide. (Note: shadow of the geographical distribution map denotes the number of EKC outbreaks in each area or country. The colours green, yellow and red denote a low, medium and high number of EKC outbreaks, respectively. The pie charts show the percentage of the serotype composition in the EKC outbreak areas or country.)

circulating throughout the world, a comprehensive analysis of ocular adenovirus infections has not been conducted. Ocular infections are largely restricted to type B and D adenoviruses (Supplementary Table S3). However, different serotypes of HAdV are associated with different disease manifestations, severity of infection, age and the underlying medical condition of the patients [3]. The present study showed that ocular HAdV serotypes were highly divergent and polymorphic in distinct geographical areas and over time, with a predominance of HAdV-8, -19 and -37 strains worldwide [62, 63].

Many ocular adenoviruses are endemic, and infections in patients in the absence of an outbreak are generally not reported due to the absence of surveillance systems in many countries. Thus, the current summary of the adenovirus-associated conjunctivitis outbreaks might be incomplete. In addition, most outbreaks 
(a)

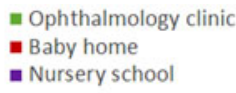

- Neonatal intensive care unit = Geriatric Centre - Plants

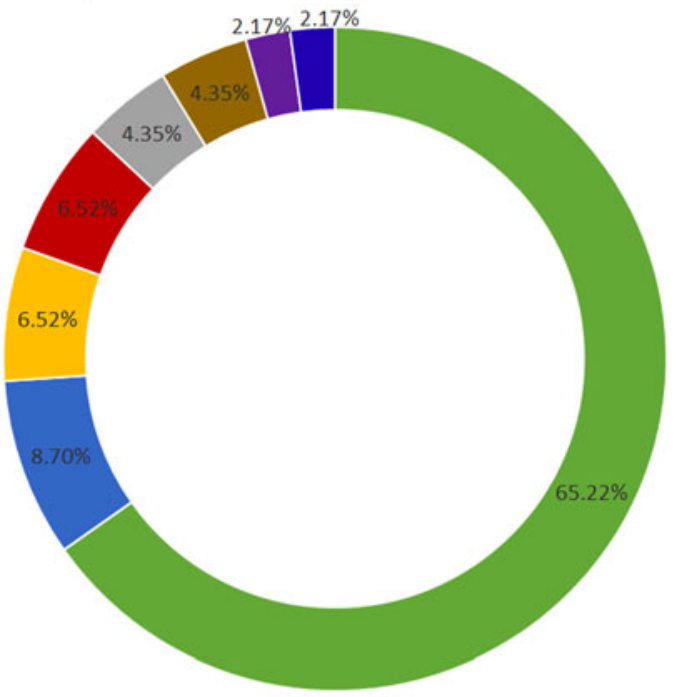

(b)

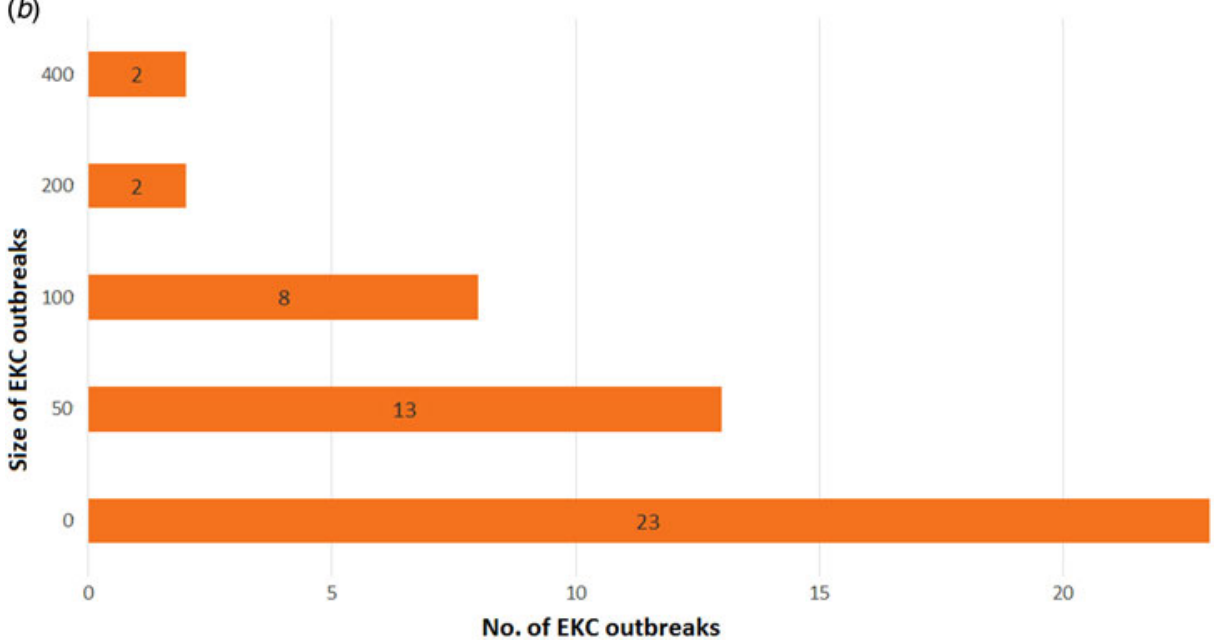

Fig. 5. (a) Locations of the 48 HAdV-associated EKC outbreaks from 1953 to 2013. (b) The distribution of the 48 HAdV-associated EKC outbreaks from 1953 to 2013.

are unreported because they are no longer novel, except when they are associated with a new type of adenovirus. The present study was initiated to describe the virology and epidemiology of reported conjunctivitis outbreaks caused by different adenovirus serotypes from 1953 to 2013, thereby extending and updating current knowledge on adenoviral-associated ocular infections. This study focused on the epidemic patterns and regularities of adenovirus associatedconjunctivitis circulating in different continents/countries, in addition to the seasonal distribution and populations affected and adenovirus subtypes.

The virological analysis supported findings in the current literature, showing that different serotypes of HAdV were associated with different types of conjunctivitis [64, 65]. The adenovirus responsible for conjunctivitis was divided into three subgroups, with no common ancestral nodes in different countries over different years, as shown by the median-joining network analysis. This result suggests that the adenoviruses maintained their overall structural features, with minimum diversity with respect to locations and transmission mechanisms. As there are different serotypes of adenoviruses in conjunctival infections, repeated infection by different variants of the virus may occur.

The hexon of HAdV has several neutralizing epitopes. Of these, the anti-hexon antibody is the most effective in neutralizing HAdV infection. To compare the structural modelling of the dominant neutralizing 
Table 1. The virology and epidemiology of the global pharyngoconjunctival fever $(P C F)$ outbreaks $(N=17)$ caused by different subtypes of human adenovirus from 1953 to 2013

\begin{tabular}{llllllll}
\hline \hline Year & Month & Country/area & Location & Age, yr & Size & Subtype & Positive rate \\
\hline 1959 & June & Austria & Victorian migrant centre & n.a. & 44 & HAdV-7 & n.a. \\
1971 & May & Australia & Melbourne & n.a. & 66 & HAdV-4 & n.a. \\
1973 & Summer & USA & Swimming pool in a junior high school & n.a. & 44 & HAdV-7 & n.a. \\
1977 & June-July & USA & A suburban community and a & n.a. & 105 & HAdV-3 & $32 \%(34 / 105)$ \\
& & & swimming pool & n.a. & 72 & HAdV-4 & $76 \cdot 92 \%(20 / 26)$ \\
1977 & Summer & USA & A private recreational facility in & & & \\
& & & Georgia & $11-18$ & 96 & HAdV-3 & n.a. \\
1981 & July & UK & A boarding school in Glasgow & $<18$ & 77 & HAdV-7a & $71 \%(5 / 7)$ \\
1982 & July & USA & A swimming pool & n.a. & 4000 & HAdV-7 & $70 \%(14 / 20)$ \\
1984 & August & China & A swimming pool in Beijing & n.a. & n.a. & HAdV-3 & n.a. \\
1991 & July & USA & A 4-week summer camp & $4-17$ & 67 & HAdV-3 & n.a. \\
1994 & July & China & A swimming pool & $<18$ & 80 & HAdV-7p & n.a. \\
1995 & July & Greece & Municipal swimming pool & n.a. & 48 & HAdV-3 & n.a. \\
2000 & October & Austria & A swimming pool & $3-6$ & 258 & HAdV-3 & $37 \%(10 / 27)$ \\
2004 & June-July & China & A swimming pool in a kindergarten & $<15$ & 134 & HAdV-3 & $100 \%(15 / 15)$ \\
2011 & June-July & China & A swimming pool & n.a. & 204 & HAdV-3 & n.a. \\
2012 & April to June & China & A swimming pool & $<15$ & 59 & HAdV-4 & $83 \cdot 33 \%(5 / 6)$ \\
2008 & June-August & Spain & Municipal swimming centre & $11-18$ & 96 & HAdV-3 & n.a. \\
1981 & July & UK & A boarding school in Glasgow & & &
\end{tabular}

n.a., Not available.

Table 2. The virology and epidemiology of the global acute haemorrhagic conjunctivitis ( AHC) outbreaks $(N=12)$ caused by different subtypes of human adenovirus from 1953 to 2013

\begin{tabular}{|c|c|c|c|c|c|c|c|}
\hline Year & Month & Country/area & Location & Age, yr & Size & Subtype & Positive rate \\
\hline 1970-1971 & May & $\begin{array}{l}\text { South-East } \\
\text { Asia }\end{array}$ & $\begin{array}{l}\text { Gandhi Memorial and } \\
\text { associated hospitals }\end{array}$ & $3-65$ & 253 & $\begin{array}{l}\text { HAdV-2 and } \\
\text { Picornavirus }\end{array}$ & n.a. \\
\hline 1973-1974 & $\begin{array}{l}\text { September- } \\
\text { October }\end{array}$ & $\begin{array}{l}\text { Japan/Sendai } \\
\text { area }\end{array}$ & $\begin{array}{l}\text { Staff of the railways and } \\
\text { their family }\end{array}$ & $5-55$ & 55 & HAdV-7, -8 & $18 \cdot 18 \%(4 / 22)$ \\
\hline 1974 & $\begin{array}{l}\text { July- } \\
\text { October }\end{array}$ & USA & Community of Seattle & n.a. & 19 & HAdV-19 & $65 \%(13 / 20)$ \\
\hline 1975 & May & USA & $\begin{array}{l}\text { Vietnamese refugees on } \\
\text { Guam }\end{array}$ & Adults & 604 & EV70 and HAdV-11 & $43 \%(43 / 100)$ \\
\hline 1982 & May-July & England & London & n.a. & 140 & $\begin{array}{l}\text { Chlamydia, HAdV, } \\
\text { and HSV }\end{array}$ & $37 \cdot 14 \%(52 / 140)$ \\
\hline 1983-1984 & $\begin{array}{r}\text { February- } \\
\text { February }\end{array}$ & Brazil & Eye clinic in Sao Paulo & n.a. & 98 & HAdV and EV70 & n.a. \\
\hline 1984 & - & China & Eye clinic & n.a. & 46 & $\begin{array}{l}\text { HAdV-3, }-7 \text { and } \\
\text { EV70 }\end{array}$ & n.a. \\
\hline 1985-1986 & February & Japan & Okinawa & n.a. & 85 & CA24v and HAdV & $45 \cdot 88 \%(39 / 85)$ \\
\hline 1985-1986 & $\begin{array}{l}\text { Winter and } \\
\text { spring }\end{array}$ & Nigeria & $\begin{array}{l}\text { Girls' boarding school and } \\
\text { local clinic }\end{array}$ & $8-19$ & 234 & HAdV-4 & $25 \%(35 / 140)$ \\
\hline 2003 & $\begin{array}{l}\text { August- } \\
\text { September }\end{array}$ & Nepal & $\begin{array}{l}\text { Tilganga Eye Centre in } \\
\text { Kathmandu }\end{array}$ & n.a. & 60 & CA24v and HAdV & $53 \cdot 33 \%(32 / 60)$ \\
\hline 2003 & $\begin{array}{l}\text { February } \\
\text { and May }\end{array}$ & Brazil & $\begin{array}{l}\text { Patients in hospital in } \\
\text { Fortaleza city }\end{array}$ & $2 \sim 66$ & 56 & CA24v and HAdV & $8 \cdot 33 \%(1 / 12)$ \\
\hline
\end{tabular}

CA24v, Coxsackie virus A24 variant; HSV, herpes simplex virus.

n.a., Not available. 
epitope functionally residing in a single capsid polypeptide, we compared the hexon's sequences and 3D structure. The ocular-associated HAdV strains shared low identity of hexon sequences, in contrast to the strains isolated from respiratory infections and their vaccine strain, which shared high identity. Ocular HAdV strains shared lower identity of hexon sequences compared to strains isolated from respiratory infections and their vaccine strain. Similarly, the 3D structure of the antigen determinant site of the hexon protein varied in different isolates from the respiratory infection, a candidate for vaccine strain and ocular infection. The configuration of the three loops was very similar in the respiratory strain and its vaccine strain, where green and yellow loops inter-related together with the red loop appeared to be more separated. In contrast, the three loops were significantly separated in the hexon protein of the ocular infection strain. The red loop was the most important epitope, it might change the overall spatial structure in this ocular strain, which is different from the other two isolates. All these sites were related to the neutralizing antibody, which induced no cross-protection in the ocular infections, even after the administration of current available vaccines against the respiratory infections [66].

There are different epidemiological features between various $\mathrm{HAdV}$-associated conjunctivitis infections. EKC due to adenovirus infection is the most common form of keratoconjunctivitis, which was supported by the findings of the present study. In the present study, the EKC outbreaks were mainly associated with HAdV-8, -19, -37 and -54, with HAdV-8 being the predominant strain over time and geographical locations. The PCF outbreaks were associated with waterborne transmission of various adenovirus types, such as HAdV-3, -4 and -7 , with HAdV-3 the most common aetiological agent. This was similar to the serotypes causing respiratory infections. The AHC outbreaks were mainly caused by HAdV-2, $-7,-8$ and -11 , and they were usually associated with co-infection with other agents. Co-infection might improve their transmission capability and result in severe ocular symptoms and signs. It is still not clear whether HAdV infection was the first pathogen.

Outbreaks of conjunctivitis caused by adenoviruses have been reported worldwide [67]. EKC and AHC are the primary forms of conjunctivitis, especially in Asia. PCF outbreaks have often been reported in tropical and subtropical areas of Australia, as well as in the United States and China. In the present study, the geographical distribution of the outbreaks was related not only to the population density and the immune status of those affected but also to the local climate, level of available healthcare and economic status. The EKC and AHC outbreaks were most often linked to ophthalmology clinics and neonatal ICU wards, whereas the sources of the PCF outbreaks were inadequately chlorinated swimming pools and small lakes in summer seasons. With regards to the chronology the outbreaks, the EKC and PCF outbreaks periodically circulated in the study populations because of the accumulation of susceptible individuals and shifts in the serotypes of the adenovirus. In contrast, many AHC outbreaks were reported in 1970 1980 , but the number of outbreaks then markedly decreased. Improvements in hygiene measures and early diagnoses may explain the decrease in the AHC outbreaks. The current study, which was based on a series of large-scale outbreaks, demonstrated that EKC was not associated with age, sex, ethnic origin, social status or nutritional status [68]. However, PCF infection was associated with young age $(<18$ years), and AHC was common in those aged 15-40 years. The different age distribution might be due to different serotypes varying in their affinity to the ocular epithelial cells, and different age groups have various protective antibody levels and also vary in their exposure risk. There are also difference in the outbreak size of EKC, PCF and AHC. Of the reported outbreaks, the AHC outbreaks were the largest, pointing to the highly contagious nature of the adenovirus responsible for this type of conjunctivitis.

Although an adenovirus vaccine has been administered to US military personnel since 1971 , this vaccine only elicits immunity to serotypes 4 and 7 . This concentred vaccine was evidenced by a reduction in the rate of febrile respiratory illness since its administration to recruits [69]. As there is currently no specific chemotherapeutic agent or broadly effective adenovirus vaccine to protect against ocular infections, strict attention to infection-control practices is likely the most effective way to prevent outbreaks of adenovirus-associated diseases, such as EKC. Additionally, maintaining adequate levels of chlorination is necessary for preventing swimming pool-associated outbreaks of adenovirus conjunctivitis. As with many other illnesses, general hygiene measures, such as hand washing, are the best way to inhibit person-to-person transmission. Improvements in surveillance systems, diagnostic and therapeutic capacities and data handling and transfer are determining factors in the control and prevention of adenovirus-induced ocular infections. Delays in the 
identification and reporting of HAdV-associated conjunctivitis outbreaks and in the institution of infectioncontrol measures can impede timely investigation and prolong transmission. It is important to establish a global adenovirus surveillance system, which should include virological and epidemiological surveillance. Such a system will aid the monitoring of adenovirus outbreaks and the detection of other unexpected novel adenoviruses that may emerge. The present study can improve our understanding of conjunctivitis and play a role in establishing a suitable strategy for the control and prevention of $\mathrm{HAdV}$-associated ocular infections.

\section{SUPPLEMENTARY MATERIAL}

For supplementary material accompanying this paper visit http://dx.doi.org/10.1017/S0950268815003246.

\section{ACKNOWLEDGEMENTS}

We are grateful to Professor Glen R. Nemerow and Professor Theresa McCarthy, Department of Immunology and Microbial Science, the Scripps Research Institute, USA for their invaluable advice in revising the manuscript.

This work was supported by the Fundamental Research Funds for the Central Universities (2015FZA7008), Technology Plan project of Zhejiang province (2012KYB090).

\section{DECLARATION OF INTEREST}

None.

\section{REFERENCES}

1. Rowe WP, et al. Isolation of a cytopathogenic agent from human adenoids undergoing spontaneous degeneration in tissue culture. Proceedings of the Society for Experimental Biology and Medicine 1953; 84: 570-573.

2. Smith JG, et al. Adenovirus. Current Topics in Microbiology and Immunology 2010; 343: 195-224.

3. Lenaerts L, et al. Clinical features and treatment of adenovirus infections. Reviews in Medical Virology 2008; 18: 357-374.

4. Robinson CM, et al. Computational analysis and identification of an emergent human adenovirus pathogen implicated in a respiratory fatality. Virology 2011; 409: 141-147.

5. Lynch 3rd JP, et al. Adenovirus. Seminars in Respiratory and Critical Care Medicine 2011; 32: 494-511.

6. O'Donnell B, et al. Genome analysis of species 3 adenoviruses isolated during summer outbreaks of conjunctivitis and pharyngoconjunctival fever in the
Glasgow and London areas in 1981. Journal of Medical Virology 1986; 18: 213-227.

7. Ford E, et al. Epidemiology of epidemic keratoconjunctivitis. Epidemiologic Reviews 1987; 9: 244-261.

8. Bialasiewicz A. Adenoviral keratoconjunctivitis. Sultan Qaboos University Medical Journal 2007; 7: 15-23.

9. Ishiko H, Aoki K. RETRACTED: Spread of epidemic keratoconjunctivitis due to a novel serotype of human adenovirus in Japan. Journal of Clinical Microbiology 2009.

10. Sundmacher R, et al. Prospects for therapy and prevention of adenovirus keratoconjunctivitis [in German]. Der Ophthalmologe 2001; 98: 991-1007; quiz 1008-1009.

11. Aoki K, Tagawa Y. A twenty-one year surveillance of adenoviral conjunctivitis in Sapporo, Japan. International Ophthalmology Clinics 2002; 42: 49-54.

12. Nakamura M, et al. Surveillance of adenovirus D in patients with epidemic keratoconjunctivitis from Fukui Prefecture, Japan, 1995-2010. Journal of Medical Virology 2012; 84: 81-86.

13. Lole KS, et al. Full-length human immunodeficiency virus type 1 genomes from subtype $\mathrm{C}$-infected seroconverters in India, with evidence of intersubtype recombination. Journal of Virology 1999; 73: 152-160.

14. Tang L, et al. Complete genome sequence of human adenovirus type 7 associated with fatal infant pneumonia. Genome Announcements 2013; 1: e00182-12.

15. Purkayastha A, et al. Genomic and bioinformatics analyses of HAdV-4vac and HAdV-7vac, two human adenovirus (HAdV) strains that constituted original prophylaxis against HAdV-related acute respiratory disease, a reemerging epidemic disease. Journal of Clinical Microbiology 2005; 43: 3083-3094.

16. Wu JS, et al. Patterns of polymorphism and divergence in the VP1 gene of enterovirus 71 circulating in the Asia-Pacific region between 1994 and 2013. Journal of Virology Methods 2013; 193: 713-728.

17. Frantzidou F, et al. Molecular epidemiology of adenovirus strains isolated from patients with ocular disease in the area of Thessaloniki, Greece (1998-2002). Journal of Medical Virology 2005; 75: 440-446.

18. Dawson C, et al. A family outbreak of adenovirus 8 infection (epidemic keratoconjunctivitis). American Journal of Hygiene 1960; 72: 279-283.

19. Richmond S, et al. A large outbreak of keratoconjunctivitis due to adenovirus type 8. Journal of Hygiene 1984; 93: $285-291$.

20. Dominguez-Berjon MF, et al. Adenovirus transmission in a nursing home: analysis of an epidemic outbreak of keratoconjunctivitis. Gerontology 2007; 53: 250-254.

21. Tullo AB, Higgins PG. An outbreak of adenovirus keratoconjunctivitis in Bristol. British Journal of Ophthalmology 1979; 63: 621-626.

22. Burns RP. Epidemic keratoconjuctivitis due to adenovirus type 19. Transactions of the Pacific Coast Oto-Ophthalmological Society Annual Meeting 1976; 57: 67-73.

23. Montessori V, et al. Epidemic keratoconjunctivitis outbreak at a tertiary referral eye care clinic. American Journal of Infection Control 1998; 26: 399-405. 
24. Novakova V, et al. Epidemic keratoconjunctivitis outbreak in a closed psychiatric ward. Infection Control and Hospital Epidemiology 2013; 34: 764-765.

25. Jin XH, et al. Genome variability of human adenovirus type 8 causing epidemic keratoconjunctivitis during 19862003 in Japan. Molecular Vision 2011; 17: 3121-3127.

26. Adlhoch $\mathbf{C}$, et al. Increasing case numbers of adenovirus conjunctivitis in Germany, 2010. Eurosurveillance 2010; 15.

27. Janani MK, et al. Isolation of a variant human adenovirus identified based on phylogenetic analysis during an outbreak of acute keratoconjunctivitis in Chennai. Indian Journal of Medical Research 2012; 136: 260-264.

28. La Rosa G, et al. Molecular characterization of human adenoviruses isolated in Italy. The New Microbiologica 2006; 29: 177-184.

29. Chastel C, et al. Molecular epidemiology of two consecutive outbreaks of adenovirus 8 keratoconjunctivitis. Journal of Medical Virology 1988; 24: 199-204.

30. Vainio K, et al. No sequence variation in part of the hexon and the fibre genes of adenovirus 8 isolated from patients with conjunctivitis or epidemic keratoconjunctivitis (EKC) in Norway during 1989 to 1996. Journal of Clinical Pathology 2001; 54: 558-561.

31. Hamada N, et al. Nosocomial outbreak of epidemic keratoconjunctivitis accompanying environmental contamination with adenoviruses. Journal of Hospital Infection 2008; 68: 262-268.

32. Ersoy Y, et al. Outbreak of adenovirus serotype 8 conjunctivitis in preterm infants in a neonatal intensive care unit. Journal of Hospital Infection 2012; 80: 144-149.

33. Melendez CP, et al. Outbreak of epidemic keratoconjunctivitis caused by adenovirus in medical residents. Molecular Vision 2009; 15: 557-562.

34. Stefkovicova M, et al. Outbreaks of epidemic keratoconjunctivitis in two hospital wards. Central European Journal of Public Health 2005; 13: 29-31.

35. Tabery HM. Two outbreaks of adenovirus type 8 keratoconjunctivitis with different outcome. Acta Ophthalmologica Scandinavica 1995; 73: 358-360.

36. Mueller AJ, Klauss V. Main sources of infection in 145 cases of epidemic keratoconjunctivitis. German Journal of Ophthalmology 1993; 2: 224-227.

37. Stasiuk RM, Robertson IF. Adeno-viral kerato conjunctivitis. Australian Journal of Ophthalmology 1981; 9: 55-58.

38. Merklova G, Saparova K. An epidemic of keratoconjunctivitis at the ophthalmic department in Levoca from October to December 1981 [in Czech]. Ceskoslovenska Oftalmologie 1985; 41: 222-224.

39. Kasova V, et al. Isolation of adenovirus type 29 from an outbreak of epidemic keratoconjunctivitis. Acta Virologica 1977; 21: 173.

40. Guyer B, et al. Epidemic keratoconjunctivitis: a community outbreak of mixed adenovirus type 8 and type 19 infection. Journal of Infectious Diseases 1975; 132: $142-150$.

41. Sprague JB, et al. Epidemic keratoconjunctivitis. A severe industrial outbreak due to adenovirus type 8 . New England Journal of Medicine 1973; 289: 1341-1346.
42. McMinn PC, et al. A community outbreak of epidemic keratoconjunctivitis in central Australia due to adenovirus type 8. Journal of Infectious Diseases 1991; 164: 1113-1118.

43. Mbonile L. Understanding of acute hemorrhagic conjunctivitis (AHC) epidemics and outbreaks of Paederus spp keratoconjuctivitis, periorbital oedema ('Nairobi red eyes') and dermatitis. East African Journal of Public Health 2010; 7: 242-245.

44. Mbonile L. Acute haemorrhagic conjunctivitis epidemics and outbreaks of Paederus spp. keratoconjunctivitis ('Nairobi red eyes') and dermatitis. South African Medical Journal 2011; 101: 541-543.

45. Melanova J, Stibor V. Epidemic of keratokonjunctivitis at the 2nd Eye Clinic in Prague during 1974-1975 [in Czech]. Ceskoslovenska oftalmologie 1976; 32: 307-310.

46. Colon LE. Keratoconjunctivitis due to adenovirus type 8: report on a large outbreak. Annals of Ophthalmology 1991; 23: 63-65.

47. Liao SH. Viral etiological study of the 1983 epidemic keratoconjunctivitis in Chengdu [in Chinese]. Chinese Journal of Ophthalmology 1987; 23: 165-167.

48. Fujii S, et al. Molecular epidemiology of adenovirus type 8 (Ad 8 ) in Taiwan: four subtypes recovered during the period of 1980-1981 from patients with epidemic keratoconjunctivitis. Japanese Journal of Medical Science \& Biology 1984; 37: 161-169.

49. Johansson ME, et al. Adenoviruses isolated in the Stockholm area during 1987-1992: restriction endonuclease analysis and molecular epidemiology. Archives of Virology 1994; 137: 101-115.

50. Salcedo Miqueleiz MA, et al. Nosocomial and community outbreak of epidemic keratoconjunctivitis in Navarra in 1996 [in Spanish]. Revista Espanola de Salud Publica 1997; 71: 383-390.

51. Aoki K, et al. An epidemic of acute hemorrhagic conjunctivitis in the city of Sao Paulo. Japanese Journal of Ophthalmology 1987; 31: 532-537.

52. Kasova $\mathbf{V}$, et al. Recurrent keratoconjunctivitis epidemic caused by adenovirus type 29 in the North Bohemia region [in Czech]. Ceskoslovenska Oftalmologie 1980; 36: 348-353.

53. Marre M, et al. Isolation and differeniation of adenovirus type 8 in epidemic keratoconjunctivitis in Leipzig [in German]. Albrecht von Graefes Archiv fur Klinische und Experimentelle Ophthalmologie. 1967; 172: 355-363.

54. Gottsch JD, et al. Prevention and control of epidemic keratoconjunctivitis in a teaching eye institute. Ophthalmic Epidemiology 1999; 6: 29-39.

55. Schrauder A, et al. Epidemic conjunctivitis in Germany, 2004. Eurosurveillance 2006; 11: 185-187.

56. Petrescu V, et al. The clinical and epidemiological aspects of a focus of epidemic keratoconjunctivitis. Revue Roumaine de Virologie (Bucharest, Romania) 1990; 41: 109-112.

57. Schepetiuk SK, et al. Outbreak of adenovirus type 4 conjunctivitis in South Australia. Journal of Medical Virology 1993; 41: 316-318.

58. Adhikary AK, et al. Serological and genetic characterisation of a unique strain of adenovirus involved in an 
outbreak of epidemic keratoconjunctivitis. Journal of Clinical Pathology 2004; 57: 411-416.

59. CDC. Epidemic keratoconjunctivitis in an ophthalmology clinic-California. Morbidity and Mortality Weekly Report 1990; 39: 598-601.

60. Huang G, et al. Outbreak of epidemic keratoconjunctivitis caused by human adenovirus type 56, China, 2012. PLOS ONE 2014; 9: e110781.

61. Chatterjee $\mathbf{S}$, et al. Unusual type of epidemic conjunctivitis in Ghana. British Journal of Ophthalmology 1970; 54: 628-630.

62. D'Angelo LJ, et al. Epidemic keratoconjunctivitis caused by adenovirus type 8: epidemiologic and laboratory aspects of a large outbreak. American Journal of Epidemiology 1981; 113: $44-49$.

63. CDC. Adenovirus-associated epidemic keratoconjunctivitis outbreaks - four states, 2008-2010. Morbidity and Mortality Weekly Report 2013; 62: 637-641.

64. Yanoff MD, Jay S. Ophthalmology 3rd edn. Mosby, 2008.
65. Adhikary AK, et al. Genetic characterisation of adenovirus type 8 isolated in Hiroshima city over a 15 year period. Journal of Clinical Pathology 2003; 56: 120-125.

66. Madisch I, et al. Phylogenetic analysis of the main neutralization and hemagglutination determinants of all human adenovirus prototypes as a basis for molecular classification and taxonomy. Journal of Virology 2005; 79: $15265-15276$.

67. Adhikary AK, Banik U. Human adenovirus type 8: the major agent of epidemic keratoconjunctivitis (EKC). Journal of Clinican Virology 2014; 61: 477-486.

68. Meyer-Rusenberg B, et al. Epidemic keratoconjunctivitis: the current situation and recommendations for prevention and treatment. Deutsches Arzteblatt International 2011; 108: 475-480.

69. Croyle MA, et al. Nasal delivery of an adenovirus-based vaccine bypasses pre-existing immunity to the vaccine carrier and improves the immune response in mice. PLOS ONE 2008; 3: e3548. 